\title{
AN INVESTIGATION OF THE GROWTH OF CHILDREN AT AN OPEN AIR SCHOOL \\ BY
}

W. H. HAMMOND

Ministry of Health, London

AND

\author{
J. A. GILLET \\ Medical Officer of Health, Dagenham, Essex
}

\begin{abstract}
Between June, 1951, and October, 1954, the growth of children attending an open air school was compared with that of children in ordinary schools.

The open air school (The Newman School), recently built and well equipped, is situated in a pleasant part of Rotherham, with its own gardens and orchard. The regime is specially designed to improve the children's general health. The children are collected daily near their homes and taken by bus to the school where they receive a light breakfast. They spend the morning doing ordinary classwork in rooms having at least one side open to the air except in extremely cold weather. When weather and other conditions allow, the children work completely in the open. There is a morning break for milk, and after a normal school lunch all the children lie down for three-quarters of an hour before beginning the afternoon's programme. This consists of a light syllabus of school work; nature walks, gardening, craft-work, singing, dancing, and physical training take up most of the time, and there is very little formal indoor class-work. At 3.30 the children have tea, consisting of milk and sandwiches or scones. They leave by bus at about 4 p.m.
\end{abstract}

\section{MATERIAL}

Children are selected to attend the Newman School for remedial treatment for a variety of physical conditions, among them general (nutritional) debility. Originally a group of 127 boys and girls aged 7-15 years who were expected to remain for at least a year was selected on nutritional grounds alone. For comparison with this group, sixty healthy children of the same ages who attended a local primary and secondary modern school, and a group of 36 brothers or sisters of the Newman children attending local schools, were selected to act as controls. These groups are referred to as the "nutritional group", "controls", and "siblings" respectively. The children's progress was followed until they left school; no new entrants were taken on. Children left the Newman School when one of us (J.A.G.), as medical officer, considered them fit to attend ordinary schools or when they reached the age of 16. As many as possible of the children who left were followed up afterwards.

Nutritional Group.-These children, regarded as undernourished and needing special care, were mainly, but not entirely, from poor homes in poor financial circumstances living in less healthy areas of the town.

Sibling Group.-This consisted of all the brothers or sisters of the "nutritional group" who were attending local schools. These children, therefore, had the same home conditions as those in the "nutritional group", but none of them appeared to be in need of special treatment on account of debility or illness. The schools they attended were, in general, less modern and in less favoured districts than the control schools.

Control Group.-This was composed of children specially selected by the medical officer and school nurses as being in good health. They attended a primary school or a secondary modern school on the same site in fairly modern buildings in a pleasant part of the town. The children were drawn mostly from new housing estates and followed the normal school routine.

\section{Procedure}

All children were measured annually in some twenty body measurements, including weight, length, girth, and breadth, and six subcutaneous fat measurements. The groups were compared with respect to physique and development. Weights were taken monthly; height, chest, and knee girths, hand breadth, inter-spinous and trochanteric breadths, and the six skinfold thickness measurements were 
taken every 3 months. The techniques have been described elsewhere (Hammond, 1953 a, b; 1955). Intelligence and scholastic tests were applied to all but the "sibling" children; school attendance and home background records were kept of sub-groups. (The mental test results are not included in the present communication.)

\section{RESULTS}

(a) Cross-Sectional Results.-Not all the measurements taken are reproduced here; those selected have been chosen with the object of covering as many aspects of physique as possible.
Table I gives the mean measurements at each age for both sexes, for weight, height, acromial breadth chest girth, body fat, and the ratios Sitting Height: Leg Length and Weight : Height. Formulae for predicting appropriate weight for a given skeletal size have been developed (Bransby and Hammond, 1951), and the actual weights relative to the expected weights are also shown. Standards of comparison consisting of the mean weights and heights of L.C.C. School Children (Daley, 1950), and body fat and other measurements (Hammond, 1953 a b, 1955) are also included.

Children mostly entered the Newman School at about the age of 8 years (except during the early

MEASUREMENTS.BY

\begin{tabular}{|c|c|c|c|c|c|c|c|c|c|c|}
\hline \multirow[b]{2}{*}{$\begin{array}{c}\text { Age } \\
\text { (yrs) }\end{array}$} & \multirow[b]{2}{*}{ Group } & \multicolumn{9}{|c|}{ BOYS } \\
\hline & & $\begin{array}{l}\text { No. in } \\
\text { Group }\end{array}$ & $\begin{array}{c}\text { Weight } \\
\text { (kg.) }\end{array}$ & $\begin{array}{r}\text { Height } \\
\text { (cm.) }\end{array}$ & $\begin{array}{c}\text { Shoulder } \\
\text { Breadth } \\
\text { (cm.). }\end{array}$ & $\begin{array}{c}\text { Chest } \\
\text { Girth } \\
\text { (Nipple) } \\
\text { (cm.) }\end{array}$ & $\begin{array}{c}\text { Body } \\
\text { Fat } \\
(0.1 \mathrm{~mm} .)\end{array}$ & $\begin{array}{c}\text { Sitting } \\
\text { Height } \\
\text { Leg Length }\end{array}$ & $\begin{array}{l}\text { Weight } \\
\text { Minus } \\
\text { Predicted } \\
\text { Weight } \\
\text { (kg.) }\end{array}$ & 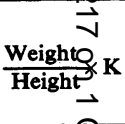 \\
\hline 7 & $\begin{array}{l}\text { Nutritional } \\
\text { Sibling } \\
\text { Controis } \\
\text { Standard }\end{array}$ & $\begin{array}{r}4 \\
2 \\
1 \\
- \\
\end{array}$ & $\begin{array}{l}21 \cdot 0 \\
23 \cdot 3 \\
27.0 \\
24 \cdot 3 \\
\end{array}$ & $\begin{array}{l}118 \cdot 5 \\
123 \cdot 3 \\
127 \cdot 4 \\
123 \cdot 2 \\
\end{array}$ & $\begin{array}{l}26.0 \\
27.8 \\
29.5 \\
27.0 \\
\end{array}$ & $\begin{array}{l}59.6 \\
58.8 \\
60.5 \\
-\end{array}$ & $\begin{array}{l}37.5 \\
33.0 \\
38.2 \\
42.5\end{array}$ & $\begin{array}{l}1 \cdot 135 \\
1 \cdot 052 \\
1 \cdot 145 \\
1 \cdot 109 \\
\end{array}$ & $\begin{array}{c}-0.1 \\
-0.04 \\
1.7 \\
-\end{array}$ & $\begin{array}{l}0.395 \\
0.415 \\
0.465 \\
0.460 \\
\end{array}$ \\
\hline 8 & $\begin{array}{l}\text { Nutritional } \\
\text { Sibling } \\
\text { Controis } \\
\text { Standard } \\
\end{array}$ & $\begin{array}{r}16 \\
6 \\
7 \\
- \\
\end{array}$ & $\begin{array}{l}22.9 * \\
23.7^{*} \\
28 \cdot 7 \\
26 \cdot 9 \\
\end{array}$ & $\begin{array}{l}125.0^{*} \\
125.5 \\
129.0 \\
127.4 \\
\end{array}$ & $\begin{array}{l}26.9 \\
27.8 \\
28.7 \\
28.0 \\
\end{array}$ & $\begin{array}{l}59 \cdot 6 \\
59 \cdot 8 \\
62 \cdot 1 \\
-\end{array}$ & $\begin{array}{l}37 \cdot 4^{*} \\
36 \cdot 0^{*} \\
61 \cdot 0^{*} \\
43 \cdot 0 \\
\end{array}$ & $\begin{array}{l}1 \cdot 062 \\
1 \cdot 061 \\
1 \cdot 080 \\
1 \cdot 072 \\
\end{array}$ & $\begin{array}{c}-1 \cdot 2^{*} \\
-1.2 \\
1.0 \\
-\end{array}$ & 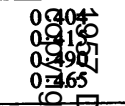 \\
\hline 9 & $\begin{array}{l}\text { Nutritional } \\
\text { Sibling } \\
\text { Controis } \\
\text { Standard }\end{array}$ & $\begin{array}{r}21 \\
6 \\
10 \\
- \\
\end{array}$ & $\begin{array}{l}25 \cdot 1^{*} \\
24 \cdot 2^{*} \\
28 \cdot 4 \\
25 \cdot 4 \\
\end{array}$ & $\begin{array}{l}130 \cdot 5 \\
129 \cdot 4 \\
130 \cdot 7 \\
133 \cdot 1\end{array}$ & $\begin{array}{l}28.1 \\
28.5 \\
28.9 \\
28.6\end{array}$ & $\begin{array}{l}62.0 \\
61.9 \\
64.2 \\
- \\
\end{array}$ & $\begin{array}{l}38.0^{*} \\
27.4^{*} \\
51.3 \\
47 \cdot 0 \\
\end{array}$ & $\begin{array}{l}1 \cdot 017 \\
1 \cdot 045 \\
1 \cdot 049 \\
1 \cdot 046 \\
\end{array}$ & $\begin{array}{c}-1 \cdot 6^{*} \\
-2 \cdot 4^{*} \\
0 \cdot 3 \\
-\end{array}$ & $\begin{array}{l}0 . \overline{4200^{*}} \\
0.422 \\
0.479 \\
0.490\end{array}$ \\
\hline 10 & $\begin{array}{l}\text { Nutritional } \\
\text { Sibling } \\
\text { Controis } \\
\text { Standard }\end{array}$ & $\begin{array}{r}25 \\
6 \\
13 \\
- \\
\end{array}$ & $\begin{array}{l}27 \cdot 2 * \\
27 \cdot 2^{*} \\
31 \cdot 5 \\
32 \cdot 1 \\
\end{array}$ & $\begin{array}{l}131.4^{*} \\
132.9 \\
134.2 \\
137.6 \\
\end{array}$ & $\begin{array}{l}28 \cdot 9 \\
29 \cdot 2 \\
30.0 \\
29 \cdot 6 \\
\end{array}$ & $\begin{array}{l}63.4 \\
64.0 \\
67.5 \\
- \\
\end{array}$ & $\begin{array}{l}37 \cdot 2 * \\
31 \cdot 2 * \\
58 \cdot 0 \\
50 \cdot 0 \\
\end{array}$ & \begin{tabular}{ll|}
$1 \cdot 010$ \\
$1 \cdot 019$ \\
$1 \cdot 020$ \\
$1 \cdot 030$ \\
\end{tabular} & $\begin{array}{l}-0.7 * \\
-2.1^{*} \\
-0.09 \\
-\end{array}$ & $\begin{array}{l}0.439 \\
0.478 \\
0.518 \\
0.528 \\
\end{array}$ \\
\hline 11 & $\begin{array}{l}\text { Nutritional } \\
\text { Sibling. } \\
\text { Controis } \\
\text { Standard } \\
\end{array}$ & $\begin{array}{r}29 \\
6 \\
13 \\
- \\
\end{array}$ & $\begin{array}{l}28 \cdot 0^{*} \\
30 \cdot 6^{*} \\
32 \cdot 9^{*} \\
34 \cdot 7\end{array}$ & $\begin{array}{l}139 \cdot 1^{*} \\
135.8^{*} \\
137.4 \\
141 \cdot 2 \\
\end{array}$ & $\begin{array}{l}29 \cdot 8 \\
28.3 \\
30.6 \\
30.0 \\
\end{array}$ & $\begin{array}{l}64.5 \\
66.0 \\
68.8 \\
- \\
\end{array}$ & $\begin{array}{l}37.9 * \\
37.4^{*} \\
55 \cdot 3 \\
52.0 \\
\end{array}$ & $\begin{array}{l}0.988 \\
1.045 \\
0.992 \\
1.005 \\
\end{array}$ & $\begin{array}{l}-3.4^{*} \\
-0.6 \\
-0.8 \\
- \\
\end{array}$ & $\begin{array}{l}0.446 \\
0.468 \\
0.527 \\
0.542\end{array}$ \\
\hline 12 & $\begin{array}{l}\text { Nutritional } \\
\text { Sibling } \\
\text { Controis } \\
\text { Standard }\end{array}$ & $\begin{array}{r}21 \\
6 \\
12 \\
\end{array}$ & $\begin{array}{l}31 \cdot 8^{*} \\
32 \cdot 2^{*} \\
35 \cdot 0^{*} \\
38 \cdot 6\end{array}$ & $\begin{array}{l}144 \cdot 1 \\
143.9 \\
143.0 \\
145.9\end{array}$ & $\begin{array}{l}30 \cdot 7 \\
31.0 \\
31.4 \\
31.0 \\
\end{array}$ & $\begin{array}{l}66.9 \\
66.7 \\
69.1 \\
- \\
\end{array}$ & $\begin{array}{l}37 \cdot 2^{*} \\
44.0^{*} \\
54 \cdot 5 \\
52 \cdot 5 \\
\end{array}$ & $\begin{array}{l}0.989 \\
0.990 \\
0.983 \\
0.981 \\
\end{array}$ & $\begin{array}{l}-3 \cdot 3^{*} \\
-1 \cdot 1 \\
-0 \cdot 6 \\
-\end{array}$ & $\begin{array}{l}0.485 \\
0.493 \\
0.549 \\
0.5 \frac{100}{2}\end{array}$ \\
\hline 13 & $\begin{array}{l}\text { Nutritional } \\
\text { Sibling } \\
\text { Controis } \\
\text { Standard }\end{array}$ & $\begin{array}{r}18 \\
8 \\
15 \\
-\end{array}$ & $\begin{array}{l}33 \cdot 6^{*} \\
34 \cdot 3^{*} \\
41 \cdot 0 \\
42 \cdot 9\end{array}$ & $\begin{array}{l}146 \cdot 5^{*} \\
147 \cdot 1^{*} \\
153.0 \\
152 \cdot 0\end{array}$ & $\begin{array}{l}31 \cdot 5 \\
31.6 \\
33.0 \\
32.0\end{array}$ & $\begin{array}{l}65.6 \\
68.4 \\
73.7 \\
-\end{array}$ & $\begin{array}{l}38 \cdot 8^{*} \\
41.0^{*} \\
53.9 \\
52 \cdot 0\end{array}$ & $\begin{array}{l}0.986 \\
0.981 \\
0.959 \\
0.955\end{array}$ & $\begin{array}{l}-2 \cdot 8^{*} \\
-2 \cdot 4 \\
-1 \cdot 0 \\
-\end{array}$ & $\begin{array}{l}0.498^{*} \\
0.519 \\
0.598 \\
0.618\end{array}$ \\
\hline 14 & $\begin{array}{l}\text { Nutritional } \\
\text { Sibling } \\
\text { Controis } \\
\text { Standard }\end{array}$ & $\begin{array}{r}18 \\
5 \\
16 \\
- \\
\end{array}$ & $\begin{array}{l}36.7 * \\
44.9 \\
46.8 \\
47.6\end{array}$ & $\begin{array}{l}152.3 \\
154.8 \\
160.5 \\
158.0 \\
\end{array}$ & $\begin{array}{l}32.9 \\
33.2 \\
34.7 \\
33.5\end{array}$ & $\begin{array}{l}71 \cdot 7 \\
72 \cdot 7 \\
76 \cdot 0 \\
- \\
\end{array}$ & $\begin{array}{l}38 \cdot 0^{*} \\
61.7 \\
50 \cdot 7 \\
51 \cdot 0 \\
\end{array}$ & $\begin{array}{l}0.986 \\
0.966 \\
0.949 \\
0.985 \\
\end{array}$ & $\begin{array}{l}-2 \cdot 9^{*} \\
-1 \cdot 5^{*} \\
=\end{array}$ & $\begin{array}{l}0.528 . \\
0.608 \\
0.659\end{array}$ \\
\hline 15 & $\begin{array}{l}\text { Nutritional } \\
\text { Sibling } \\
\text { Controis } \\
\text { Standard }\end{array}$ & $\begin{array}{r}5 \\
2 \\
6 \\
- \\
\end{array}$ & $\begin{array}{l}39 \cdot 0^{*} \\
62 \cdot 2^{*} \\
50 \cdot 1 \\
54 \cdot 9 \\
\end{array}$ & $\begin{array}{l}156.6 \\
164.3 \\
165.4 \\
165.7 \\
\end{array}$ & $\begin{array}{l}33 \cdot 8 \\
33.8 \\
35.5 \\
35 \cdot 0 \\
\end{array}$ & $\begin{array}{l}73.4 \\
66.5 \\
78.9 \\
71.0 \\
\end{array}$ & $\begin{array}{c}37 \cdot 7 * \\
110 \cdot 5^{*} \\
50 \cdot 5 \\
50.0\end{array}$ & $\begin{array}{l}0.952 \\
0.994 \\
0.873 \\
-\end{array}$ & $\begin{array}{l}-4 \cdot 2 * \\
11 \cdot 1^{*} \\
-0 \cdot 8 \\
-\end{array}$ & $\begin{array}{l}0.5480 \\
0.739 \\
0.667 \\
0.790\end{array}$ \\
\hline 16 & $\begin{array}{l}\text { Nutritional } \\
\text { Sibling } \\
\text { Controis } \\
\text { Standard } \\
\end{array}$ & $\frac{1}{1}$ & $\begin{array}{l}41.9 \\
57.6 \\
60.8\end{array}$ & $\begin{array}{l}162 \cdot 5 \\
168 \cdot 0 \\
172 \cdot 0\end{array}$ & $\begin{array}{l}36 \cdot 5 \\
3 \overline{36 \cdot 0} \\
36 \cdot 0\end{array}$ & $\begin{array}{l}78 \cdot 0 \\
83 \cdot 5 \\
82 \cdot 0\end{array}$ & $\begin{array}{l}37 \cdot 0 * \\
60 \cdot 9 \\
54 \cdot 5\end{array}$ & $\begin{array}{l}0.909 \\
0.944 \\
-\end{array}$ & $\frac{-6 \cdot 0^{*}}{1 \cdot 6^{*}}$ & $\begin{array}{l}0.565 \\
0.68 \\
0.728\end{array}$ \\
\hline 17 & Nutritional & - & - & - & - & - & - & - & - & क \\
\hline
\end{tabular}


years when the school was being established); hence children first measured at the older ages had attended the school longer than the younger children. Results for the 3 years of the study were analysed separately, but no consistent differences between them were found. The third-year measurements for age group 7 coincided very closely with the second-year measurements for age group 8 and the first-year measurements for age group 9, and these have therefore been combined.

The "nutritional" group had lower than average weight, height, and body fat at all ages. Their weights for the corresponding heights were also lower than average, but the ratio of trunk to leg length, which tends to decrease with age and reflects the changing proportions with maturation, was actually lower than the age norms, which suggests that these children were certainly not retarded in maturation. The measurement of children in the "sibling" group showed a similar inferiority, but the girls were nearer the norms. The "control" girls were well above the standards but only the younger boys were above. The results, so far, agree with what we would expect from the method of selection of the groups, and they show that the different physical criteria agree as a whole with the clinical assessment. Even the skeletal measurements, which are only influenced by fairly long-term effects, also showed the group

SEX AND GROUP

\begin{tabular}{|c|c|c|c|c|c|c|c|c|}
\hline \multicolumn{9}{|c|}{ GIRLS } \\
\hline $\begin{array}{l}\text { No. in } \\
\text { Group }\end{array}$ & $\begin{array}{c}\text { Weight } \\
\text { (kg.) }\end{array}$ & $\begin{array}{l}\text { Height } \\
\text { (cm.) }\end{array}$ & $\begin{array}{c}\text { Shoulder } \\
\text { Breadth } \\
\text { (cm.) }\end{array}$ & $\begin{array}{c}\text { Chest } \\
\text { Girth } \\
\text { (Xiphoid) } \\
\text { (cm.) }\end{array}$ & $\begin{array}{c}\text { Body } \\
\text { Fat } \\
(0.1 \mathrm{~mm} .)\end{array}$ & $\frac{\begin{array}{c}\text { Sitting } \\
\text { Height }\end{array}}{\text { Leg Length }}$ & $\begin{array}{c}\text { Weight } \\
\text { minus } \\
\text { Predicted } \\
\text { Weight } \\
\text { (kg.) }\end{array}$ & $\frac{\text { Weight }}{\text { Height }} \times \mathrm{K}$ \\
\hline $\begin{array}{r}7 \\
1 \\
1 \\
-\end{array}$ & $\begin{array}{l}18 \cdot 6^{*} \\
19 \cdot 4 \\
24 \cdot 9 \\
23 \cdot 3\end{array}$ & $\begin{array}{l}112 \cdot 3^{*} \\
118 \cdot 2 \\
121.6 \\
121 \cdot 6\end{array}$ & $\begin{array}{l}25 \cdot 2 \\
25 \cdot 5 \\
27 \cdot 0 \\
26 \cdot 0\end{array}$ & $\begin{array}{l}55 \cdot 4 \\
51 \cdot 0 \\
57.5 \\
56 \cdot 5\end{array}$ & $\begin{array}{c}43 \cdot 8 \\
41 \cdot 5 \\
(57) \\
50 \cdot 5\end{array}$ & $\begin{array}{l}1.067 \\
1.087 \\
1.068 \\
1.089\end{array}$ & $\begin{array}{c}-0.6 \\
-1.8^{*} \\
0.04 \\
-\end{array}$ & $\begin{array}{l}0.365 \\
0.361 \\
0.451 \\
0.423\end{array}$ \\
\hline $\begin{array}{r}13 \\
4 \\
6 \\
-\end{array}$ & $\begin{array}{l}21 \cdot 6^{*} \\
23 \cdot 1^{*} \\
30 \cdot 0 \\
25 \cdot 8\end{array}$ & $\begin{array}{l}120 \cdot 6^{*} \\
123 \cdot 6^{2} \\
129 \cdot 3 \\
126 \cdot 1\end{array}$ & $\begin{array}{l}26 \cdot 8 \\
27 \cdot 0 \\
29 \cdot 0 \\
27 \cdot 0\end{array}$ & $\begin{array}{l}56 \cdot 5 \\
56 \cdot 5 \\
50 \cdot 1 \\
58 \cdot 0\end{array}$ & $\begin{array}{l}49.9 \\
59.6 \\
91.0 \\
60.0\end{array}$ & $\begin{array}{l}1.033 \\
1.088 \\
1.077 \\
1.068\end{array}$ & $\begin{array}{c}-0 \cdot 1 \\
-0 \cdot 2 \\
1 \cdot 8^{*} \\
-\end{array}$ & $\begin{array}{l}0.397 \\
0.412 \\
0.513 \\
0.450\end{array}$ \\
\hline $\begin{array}{r}17 \\
5 \\
9 \\
-\end{array}$ & $\begin{array}{l}23 \cdot 3^{*} \\
24 \cdot 8^{*} \\
31 \cdot 2^{*} \\
28 \cdot 6\end{array}$ & $\begin{array}{l}125 \cdot 0^{*} \\
127 \cdot 5 \\
133 \cdot 7 \\
130.7\end{array}$ & $\begin{array}{l}27 \cdot 79 \\
28 \cdot 30 \\
31 \cdot 92 \\
28 \cdot 0\end{array}$ & $\begin{array}{l}57 \cdot 3 \\
57 \cdot 8 \\
61 \cdot 2 \\
60 \cdot 0\end{array}$ & $\begin{array}{l}52.9 \\
55.9 \\
80.3 \\
63.0\end{array}$ & $\begin{array}{l}1.028 \\
1.030 \\
1.037 \\
1.040\end{array}$ & $\begin{array}{c}-0.7 \\
-1 \cdot 1 \\
1.9^{*} \\
-\end{array}$ & $\begin{array}{l}0.410 \\
0.429 \\
0.509 \\
0.480\end{array}$ \\
\hline $\begin{array}{r}29 \\
6 \\
12 \\
-\end{array}$ & $\begin{array}{l}25 \cdot 9^{*} \\
28 \cdot 2^{*} \\
33 \cdot 7^{*} \\
31 \cdot 5\end{array}$ & $\begin{array}{l}130 \cdot 8^{*} \\
131 \cdot 4 \\
139 \cdot 2 \\
136 \cdot 1\end{array}$ & $\begin{array}{l}28 \cdot 36 \\
29 \cdot 33 \\
33 \cdot 04 \\
29 \cdot 0\end{array}$ & $\begin{array}{l}58 \cdot 9 \\
60 \cdot 0 \\
62 \cdot 2 \\
61 \cdot 0\end{array}$ & $\begin{array}{l}54 \cdot 5 \\
67 \cdot 8 \\
80 \cdot 0 \\
54 \cdot 0\end{array}$ & $\begin{array}{l}1.015 \\
1.045 \\
1.015 \\
1.028\end{array}$ & $\begin{array}{c}-1 \cdot 1^{*} \\
0.2 \\
0.4 \\
-\end{array}$ & $\begin{array}{l}0.432 \\
0.471 \\
0.532 \\
0.506\end{array}$ \\
\hline $\begin{array}{r}30 \\
7 \\
17 \\
-\end{array}$ & $\begin{array}{l}29 \cdot 1^{*} \\
32 \cdot 7^{*} \\
32 \cdot 5^{*} \\
35 \cdot 8\end{array}$ & $\begin{array}{l}137 \cdot 3^{*} \\
139 \cdot 1 \\
140 \cdot 9 \\
142 \cdot 6\end{array}$ & $\begin{array}{l}31 \cdot 31 \\
30 \cdot 57 \\
31 \cdot 59 \\
30 \cdot 0\end{array}$ & $\begin{array}{l}61 \cdot 2 \\
62 \cdot 5 \\
65 \cdot 5 \\
64 \cdot 0\end{array}$ & $\begin{array}{l}53 \cdot 6 \\
78 \cdot 3 \\
68 \cdot 8 \\
69 \cdot 0\end{array}$ & $\begin{array}{l}1.014 \\
0.973 \\
1.009 \\
1.010\end{array}$ & $\begin{array}{l}-2.4^{*} \\
-0.5 \\
-0.8 \\
-\end{array}$ & $\begin{array}{l}0.470 \\
0.520 \\
0.542 \\
0.552\end{array}$ \\
\hline $\begin{array}{r}28 \\
9 \\
17 \\
-\end{array}$ & $\begin{array}{l}32 \cdot 6^{*} \\
33 \cdot 8^{*} \\
36 \cdot 9^{*} \\
40 \cdot 4^{*}\end{array}$ & $\begin{array}{l}143 \cdot 8 \\
144 \cdot 9 \\
147 \cdot 6 \\
148 \cdot 5\end{array}$ & $\begin{array}{l}31 \cdot 23 \\
30 \cdot 61 \\
32 \cdot 53 \\
31 \cdot 0\end{array}$ & $\begin{array}{l}63 \cdot 6 \\
61 \cdot 8 \\
65 \cdot 5 \\
66 \cdot 0\end{array}$ & $\begin{array}{l}55 \cdot 7 \\
55 \cdot 5 \\
73 \cdot 8 \\
74 \cdot 0\end{array}$ & $\begin{array}{l}0.994 \\
0.969 \\
1.008 \\
1.003\end{array}$ & $\begin{array}{l}-2.1^{*} \\
-0.9 \\
-0.5 \\
-\end{array}$ & $\begin{array}{l}0.497 \\
0.515 \\
0.550 \\
0.596\end{array}$ \\
\hline $\begin{array}{r}29 \\
8 \\
16 \\
-\end{array}$ & $\begin{array}{l}37 \cdot 2^{*} \\
39 \cdot 5^{*} \\
47 \cdot 6^{*} \\
45 \cdot 0^{*}\end{array}$ & $\begin{array}{l}149 \cdot 5^{*} \\
150 \cdot 2 \\
156 \cdot 4 \\
153 \cdot 1\end{array}$ & $\begin{array}{l}32 \cdot 52 \\
32 \cdot 12 \\
34 \cdot 16 \\
32 \cdot 0\end{array}$ & $\begin{array}{l}64 \cdot 9 \\
64 \cdot 8 \\
67 \cdot 19 \\
67 \cdot 0\end{array}$ & $\begin{array}{l}62 \cdot 2 \\
67 \cdot 3 \\
87 \cdot 9 \\
88 \cdot 0\end{array}$ & $\begin{array}{l}0.994 \\
1.033 \\
1.000 \\
1.007\end{array}$ & $\begin{array}{l}-1.4^{*} \\
-0.9 \\
-0.9 \\
-\end{array}$ & $\begin{array}{l}0.547 \\
0.578 \\
0.670 \\
0.646\end{array}$ \\
\hline $\begin{array}{r}23 \\
3 \\
15 \\
-\end{array}$ & $\begin{array}{l}40 \cdot 5^{*} \\
48 \cdot 3 \\
50 \cdot 2 \\
49 \cdot 2\end{array}$ & $\begin{array}{l}153.6 \\
153.9 \\
158.6 \\
150.9\end{array}$ & $\begin{array}{l}33.04 \\
34.0 \\
35.0 \\
34.0\end{array}$ & $\begin{array}{l}66 \cdot 4 \\
66 \cdot 8 \\
69 \cdot 3 \\
69 \cdot 0\end{array}$ & $\begin{array}{l}61 \cdot 0 \\
97 \cdot 3 \\
94 \cdot 7 \\
96.0\end{array}$ & $\begin{array}{l}0.987 \\
1.062 \\
0.986 \\
1.016\end{array}$ & $\begin{array}{c}-3 \cdot 5^{*} \\
2 \cdot 7^{*} \\
-2 \cdot 0^{*} \\
-\end{array}$ & $\begin{array}{l}0.560 \\
0.669 \\
0.698 \\
0.692\end{array}$ \\
\hline $\begin{array}{r}7 \\
3 \\
5 \\
-\end{array}$ & $\begin{array}{c}37 \cdot 1^{*} \\
(47 \cdot 3) \\
51 \cdot 0 \\
52 \cdot 2\end{array}$ & $\begin{array}{l}148 \cdot 0^{*} \\
159 \cdot 0 \\
156 \cdot 7 \\
162 \cdot 5\end{array}$ & $\begin{array}{l}32 \cdot 5 \\
34 \cdot 25 \\
34 \cdot 7 \\
34 \cdot 0\end{array}$ & $\begin{array}{l}64 \cdot 5 \\
68 \cdot 7 \\
72 \cdot 5 \\
70 \cdot 0\end{array}$ & $\begin{array}{r}62 \cdot 1 \\
94 \cdot 5 \\
104 \cdot 1 \\
100 \cdot 0\end{array}$ & $\begin{array}{c}1.044 \\
1.006 \\
1.018 \\
-\end{array}$ & $\begin{array}{r}-1.5 \\
-0.5 \\
0.2 \\
-\end{array}$ & $\begin{array}{l}0.546 \\
0.656 \\
0.718 \\
0.705\end{array}$ \\
\hline $\begin{array}{r}2 \\
1 \\
-\end{array}$ & $\begin{array}{c}\left(36 \cdot 5^{*}\right) \\
\left(45 \cdot 3^{*}\right) \\
5 \overline{5} \cdot 0\end{array}$ & $\begin{array}{l}145 \cdot 6^{*} \\
159 \cdot 2 \\
16 \overline{7 \cdot 5}\end{array}$ & $\begin{array}{l}30 \cdot 2 \\
36 \cdot 0 \\
3 \overline{4} \cdot 0\end{array}$ & $\begin{array}{l}65 \cdot 7 \\
67 \cdot 5 \\
71 \cdot 0\end{array}$ & $\begin{array}{c}70 \cdot 7 \\
105 \cdot 0 \\
=\end{array}$ & $\begin{array}{c}1 \cdot 039 \\
= \\
=\end{array}$ & $\begin{array}{c}2 \cdot 1 \\
-3 \cdot 4^{*} \\
-\end{array}$ & $\begin{array}{c}0.553 \\
0.628 \\
=\end{array}$ \\
\hline 2 & $43 \cdot 1$ & $154 \cdot 4$ & $31 \cdot 8$ & $67 \cdot 2$ & $107 \cdot 0$ & - & $-2 \cdot 7^{*}$ & 0.613 \\
\hline
\end{tabular}


TABLE II

PERCENTAGE OF MEASUREMENTS ABOVE AGE STANDARDS IN DIFFERENT PHYSICAL CRITERIA

\begin{tabular}{|c|c|c|c|c|c|c|c|c|}
\hline \multirow[b]{2}{*}{ Sex } & \multirow[b]{2}{*}{ Group } & \multirow[b]{2}{*}{ No. in Group } & \multicolumn{6}{|c|}{ Measurement } \\
\hline & & & Weight & Height & $\begin{array}{l}\text { Chest } \\
\text { Girth }\end{array}$ & $\begin{array}{c}\text { Shoulder } \\
\text { Breadth }\end{array}$ & Body Fat & $\begin{array}{l}\text { Weight minus } \\
\text { Predicted } \\
\text { Weight }\end{array}$ \\
\hline Boys & $\begin{array}{l}\text { Nutritional } \\
\text { Sibling } \\
\text { Control }\end{array}$ & $\begin{array}{r}159 \\
47 \\
92\end{array}$ & $\begin{array}{r}7 \\
5 \\
44\end{array}$ & $\begin{array}{l}28 \\
23 \\
47\end{array}$ & $\begin{array}{l}17 \\
18 \\
51\end{array}$ & $\begin{array}{l}34 \\
47 \\
52\end{array}$ & $\begin{array}{r}7 \\
7 \\
50\end{array}$ & $\begin{array}{r}9 \\
14 \\
41\end{array}$ \\
\hline Girls & $\begin{array}{l}\text { Nutritional } \\
\text { Sibling } \\
\text { Control }\end{array}$ & $\begin{array}{r}179 \\
51 \\
97\end{array}$ & $\begin{array}{l}12 \\
24 \\
51\end{array}$ & $\begin{array}{l}30 \\
23 \\
53\end{array}$ & $\begin{array}{l}29 \\
26 \\
53\end{array}$ & $\begin{array}{l}43 \\
55 \\
75\end{array}$ & $\begin{array}{l}20 \\
52 \\
70\end{array}$ & $\begin{array}{l}26 \\
37 \\
53\end{array}$ \\
\hline
\end{tabular}

differentiation, suggesting that the "nutritional" group inferiority (whether due partly or wholly to nutritional influences) had been operating for some time previously.

"IDEAL" WeIGHTS.-All children of a given age cannot be expected to have the same weight, even if they have the same height, and a rather more complicated function than the weight for height ratio was used throughout in order to make allowance for the differences in skeletal framework between the groups. The regression of weight on height, shoulder (acromial) breadth, hip (trochanteric) breadth, and hand breadth, was used to calculate the expected weight in relation to these measurements, and any difference between the actual weight and the calculated weight would be due to other parts, chiefly muscle and fat. The differences between actual and expected weights are shown in Column 7 of Table I. In general, the results are very similar to those for weight according to height, the "nutritional" and "sibling" groups being low in weight even compared with their smaller skeletal framework, and the "controls" showing an excess of weight. What applies to the group does not necessarily apply to each individual, and Table II shows the percentage of children in each group above the standards.* (The results for the 3 years are combined.)

The percentages reaching the standards for each age were not greatly different for each of the years 1951-54, except in the nutritional group in which the numbers above average in weight rose from 8 to 19 per cent., and above average in height from 16 to 23 per cent. for girls, and from 23 to 36 per cent. for boys. Body fat was the single criterion which agreed best with the group selection, since only

*50 per cent. is the expected value for a normal distribution if the mean is equal to the standard. In children's weight and body fat measurements which are skewed, $\mathbf{4 0}$ per cent. of children were above the means (unpublished data). This percentage was sufficient to balance the 60 per cent. below standard, since positive deviations can be much higher than negative ones.
13 per cent. of the "nutritional" children reached the standard, whilst over 60 per cent. of "controls" reached or exceeded it. Attained weight gave the next closest agreement.

Physical TyPe.-This is largely a constitutional matter but, as measured by the relation of girth measurements to linear (bone) measurements, it is also influenced by environment. $\dagger$

The nutritional group contained the most leptosomes (long, thin type), the controls contained most eurysomes (stocky, muscular or fat types), and the siblings were about equally divided.

\begin{tabular}{lll|c|c}
\hline \multirow{2}{*}{ Group } & & \multicolumn{2}{c}{ Percentage } \\
\cline { 5 - 5 } & & & Leptosomes & Eurysomes \\
\hline Nutritional &.. &. & 63 & 37 \\
Sibling.. &. &. & 49 & 51 \\
Control &.. &.. & 41 & 59 \\
\hline
\end{tabular}

This association between physical type and nutritional grouping could mean either that the initial selection of the children took into account physique as one factor in the clinical appraisal, so that the leptosomes being thinner were more often downgraded than the other types, or that the leptosome type is more prone to suffer from general debility (See also Bransby and Hammond, 1951). In fact, however, the actual type measurements were not known at the time of selection.

(b) Longitudinal Results.-As some children left during the study it was not possible to use the crosssectional results to give any precise information on the growth changes in the different groups. The actual growth in weight and height has therefore been analysed. Growth differs much less than

tThe type "scores" were determined as weighted sums of length and breadth measurements, the weights being the regression coefficients breadth measurements, the weights being the regression coeficients
between the measurement and the type distinction as previously derived from factor analyses of children's measurements (Hammond, 1953 b). Children were then classified as leptosomes or eurysomes according to their relation to the average values for age and sex. 
TABLE III

MEAN ANNUAL GAINS IN WEIGHT AND HEIGHT FROM 1951 TO 1954

\begin{tabular}{|c|c|c|c|c|c|c|c|c|c|}
\hline \multirow{2}{*}{ Year } & \multirow{2}{*}{ Group } & \multicolumn{4}{|c|}{ Boys } & \multicolumn{4}{|c|}{ Girls } \\
\hline & & $\begin{array}{l}\text { No. in } \\
\text { Group }\end{array}$ & $\underset{\text { (yrs) }}{\text { Mean Age }}$ & $\underset{\text { (kg.) }}{\text { Weight Growth }}$ & $\begin{array}{c}\text { Height Growth } \\
\text { (cm.) }\end{array}$ & $\begin{array}{l}\text { No. in } \\
\text { Group }\end{array}$ & $\underset{\text { (yrs) }}{\text { Mean Age }}$ & $\underset{\text { (kg.) }}{\text { Weight Growth }}$ & \begin{tabular}{|} 
Height Growth \\
(cm.)
\end{tabular} \\
\hline $1951-52$ & $\begin{array}{ll}\text { Nutritional } & \ldots \\
\text { Sibling . } & \ldots \\
\text { Control . } & \cdots \\
\text { L.C.C. Standard }\end{array}$ & $\begin{array}{l}40 \\
10 \\
28\end{array}$ & $\begin{array}{l}10 \cdot 5 \\
10 \cdot 7 \\
11 \cdot 0 \\
10 \cdot 5\end{array}$ & $\begin{array}{l}2 \cdot 54 \pm 0 \cdot 21 \\
2 \cdot 75 \pm 0 \cdot 54 \\
3 \cdot 45 \pm 0 \cdot 39 \\
3 \cdot 55\end{array}$ & $\begin{array}{l}5 \cdot 78 \pm 0 \cdot 23 \\
5 \cdot 45 \pm 0 \cdot 30 \\
5 \cdot 79 \pm 0 \cdot 29 \\
5 \cdot 2\end{array}$ & $\begin{array}{l}45 \\
13 \\
29\end{array}$ & $\begin{array}{l}10 \cdot 8 \\
10 \cdot 5 \\
11 \cdot 1 \\
11 \cdot 0\end{array}$ & $\begin{array}{l}3 \cdot 00 \pm 0 \cdot 18 \\
3 \cdot 59 \pm 0 \cdot 48 \\
4 \cdot 59 \pm 0 \cdot 31 \\
3 \cdot 82\end{array}$ & $\begin{array}{l}5 \cdot 77 \pm 0 \cdot 21 \\
5 \cdot 48 \pm 0 \cdot 71 \\
5 \cdot 12 \pm 0 \cdot 31 \\
5 \cdot 4\end{array}$ \\
\hline $1952-53$ & $\begin{array}{ll}\text { Nutritional } & \ldots \\
\text { Sibling . . } & \cdots \\
\text { Control . . } & \cdots \\
\text { L.C.C. Standard }\end{array}$ & $\begin{array}{r}31 \\
9 \\
22\end{array}$ & $\begin{array}{l}10 \cdot 7 \\
10 \cdot 9 \\
11 \cdot 6 \\
11 \cdot 0\end{array}$ & $\begin{array}{l}2 \cdot 82 \pm 0 \cdot 18 \\
2 \cdot 90 \pm 0 \cdot 39 \\
4 \cdot 80 \pm 0 \cdot 28 \\
3 \cdot 77\end{array}$ & $\begin{array}{l}5 \cdot 42 \pm 0 \cdot 28 \\
5 \cdot 47 \pm 0 \cdot 33 \\
6 \cdot 14 \pm 0 \cdot 36 \\
5 \cdot 4\end{array}$ & $\begin{array}{l}39 \\
10 \\
20\end{array}$ & $\begin{array}{l}11 \cdot 4 \\
11 \cdot 5 \\
11 \cdot 6 \\
11 \cdot 5\end{array}$ & $\begin{array}{l}4 \cdot 20 \pm 0 \cdot 31 \\
3 \cdot 52 \pm 0 \cdot 75 \\
4 \cdot 24 \pm 0 \cdot 64 \\
4 \cdot 05\end{array}$ & $\begin{array}{l}6 \cdot 10 \pm 0 \cdot 33 \\
4 \cdot 68 \pm 0 \cdot 64 \\
6 \cdot 19 \pm 0 \cdot 48 \\
5 \cdot 3\end{array}$ \\
\hline 1953-54 & \begin{tabular}{ll|} 
Nutritional & $\cdots$ \\
Sibling . . & $\cdots$ \\
Control & $\cdots$ \\
L.C.C. Standard
\end{tabular} & $\begin{array}{r}23 \\
6 \\
15\end{array}$ & $\begin{array}{l}11 \cdot 6 \\
12 \cdot 0 \\
12 \cdot 8 \\
12 \cdot 0\end{array}$ & $\begin{array}{l}3 \cdot 65 \pm 0 \cdot 30 \\
4 \cdot 49 \pm 1 \cdot 04 \\
5 \cdot 08 \pm 0 \cdot 60 \\
4 \cdot 23\end{array}$ & $\begin{array}{l}5 \cdot 57 \pm 0 \cdot 37 \\
6 \cdot 03 \pm 0 \cdot 75 \\
6 \cdot 00 \pm 0 \cdot 45 \\
5 \cdot 35\end{array}$ & $\begin{array}{r}28 \\
7 \\
13\end{array}$ & $\begin{array}{l}12 \cdot 0 \\
11 \cdot 5 \\
11 \cdot 7 \\
12 \cdot 0\end{array}$ & $\begin{array}{l}4 \cdot 31 \pm 0 \cdot 41 \\
3 \cdot 85 \pm 0 \cdot 32 \\
5 \cdot 83 \pm 0 \cdot 75 \\
4 \cdot 13\end{array}$ & $\begin{array}{l}5 \cdot 94 \pm 0.47 \\
5 \cdot 51 \pm 0.59 \\
4 \cdot 29 \pm 0.56 \\
5 \cdot 2\end{array}$ \\
\hline
\end{tabular}

TABLE IV

PERCENTAGE OF CHILDREN WITH GROWTH GREATER THAN AGE STANDARDS

\begin{tabular}{|c|c|c|c|c|c|c|c|c|c|c|}
\hline \multirow{2}{*}{\multicolumn{3}{|c|}{ Group }} & \multicolumn{4}{|c|}{ Boys } & \multicolumn{4}{|c|}{ Girls } \\
\hline & & & $\begin{array}{l}\text { Number of } \\
\text { Measurements }\end{array}$ & Weight & Height & $\begin{array}{c}\text { Body } \\
\text { Fat }\end{array}$ & $\begin{array}{l}\text { Number of } \\
\text { Measurements }\end{array}$ & Weight & Height & $\begin{array}{l}\text { Body } \\
\text { Fat }\end{array}$ \\
\hline $\begin{array}{l}\text { Nutritional } \\
\text { Sibling . } \\
\text { Control }\end{array}$ & $\begin{array}{l}\ddot{*} \\
\ddot{*}\end{array}$ & $\begin{array}{l}\cdots \\
\cdots \\
\cdots\end{array}$ & $\begin{array}{l}94 \\
25 \\
65\end{array}$ & $\begin{array}{l}25 \\
18 \\
41\end{array}$ & $\begin{array}{l}52 \\
42 \\
55\end{array}$ & $\begin{array}{l}61 \\
55 \\
55\end{array}$ & $\begin{array}{r}112 \\
30 \\
62\end{array}$ & $\begin{array}{l}40 \\
50 \\
61\end{array}$ & $\begin{array}{l}58 \\
53 \\
38\end{array}$ & $\begin{array}{l}50 \\
68 \\
64\end{array}$ \\
\hline
\end{tabular}

attained measurements from one age to another (except at the pubertal spurt) and hence the growth in weight and height has been combined for all ages of children in the three groups. The mean ages rarely differed by more than a few months. The weight and height increases and their standard errors for each of the 3 years of the study, are set out in Table III, together with the mean ages of the groups and the annual weight and height differences for L.C.C. children in these age ranges (Daley, 1950). Table IV gives the percentage of children whose growth in weight and height and change in body fat was greater than their age standards.

In general, only the "control" group had weight gains consistently above the standard L.C.C. differences, but for all groups of boys, and also for the girls of the "nutritional" group after the first year, the mean height growth was greater than the L.C.C. differences.

There was no marked change relative to the L.C.C. figures during the course of the study, although more children of all groups gained body fat at a greater rate than the standard's change with age, especially towards the end of the study. Over the 3 years the "control" boys gained most weight and height, whilst the "control" girls gained most weight but not height (some tending to put on too much fat).

These growth results are more favourable to the "nutritional" groups than are the cross-sectional results, but although their height gain exceeded that of L.C.C. children, the weight gain did not keep pace.

Weight Growth in Relation to Height GrowTH.-Growth in weight and height may be plotted against each other and it is then found that the average relationship tends to have the same slope for all years within the following age ranges:

Boys 7-12 and 13-15 years;

Girls 7-10, 11-12, 13-16 years.

Over these ages, therefore, we can compare the number of children within the "nutritional", "sibling", or "control" groups whose weight gain relative to their height gain for age is greater or less than the average, and these children may then be combined over the whole age range. The results are shown in Table V.

TABLE V

RELATION BETWEEN GAINS IN WEIGHT AND HEIGHT

\begin{tabular}{|c|c|c|c|c|c|}
\hline \multirow[t]{2}{*}{ Sex } & \multirow{2}{*}{\multicolumn{2}{|c|}{ Group }} & \multirow[t]{2}{*}{$\begin{array}{l}\text { No. in } \\
\text { Group }\end{array}$} & \multicolumn{2}{|c|}{$\begin{array}{l}\text { Percentage with } \\
\text { Weight Gain related } \\
\text { to Height Gain }\end{array}$} \\
\hline & & & & High & Low \\
\hline Boys & $\begin{array}{l}\text { Nutritional } \\
\text { Sibling } \quad \ldots \\
\text { Control } \quad .\end{array}$ & $\begin{array}{l}\cdots \\
\cdots \\
\cdots\end{array}$ & $\begin{array}{l}89 \\
27 \\
51\end{array}$ & $\begin{array}{l}43 \\
61 \\
64\end{array}$ & $\begin{array}{l}57 \\
39 \\
36\end{array}$ \\
\hline Girls & $\begin{array}{l}\text { Nutritional } \\
\text { Sibling } \quad \ldots \\
\text { Control } \quad \ldots\end{array}$ & $\begin{array}{l}\cdots \\
\cdots\end{array}$ & $\begin{array}{r}112 \\
65 \\
25\end{array}$ & $\begin{array}{l}41 \\
60 \\
66\end{array}$ & $\begin{array}{l}59 \\
40 \\
34\end{array}$ \\
\hline
\end{tabular}



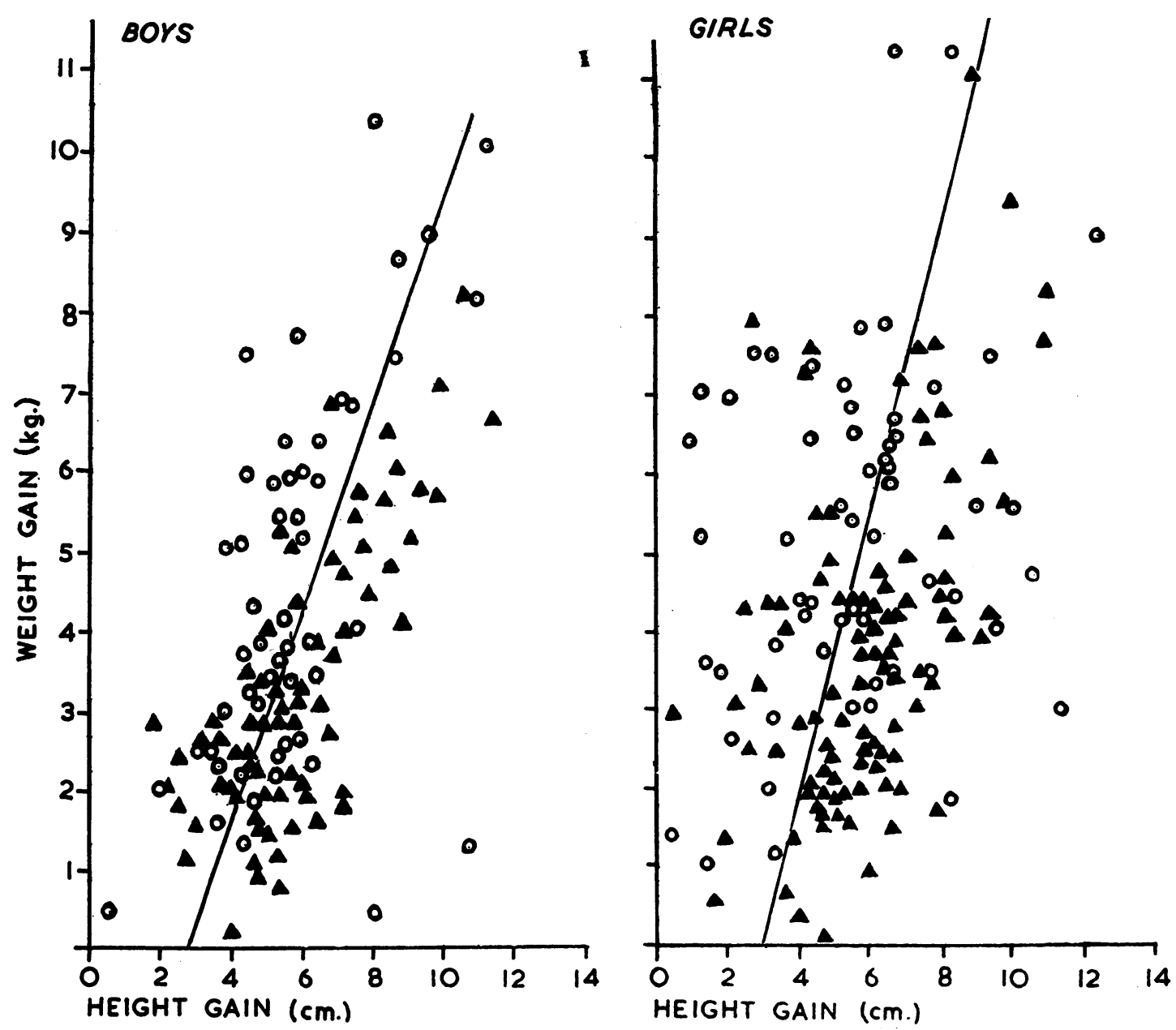

FIGURE.-Annual weight gain according to height gain in boys and girls.

The Figure shows that not only do the "control" children (o) gain more weight and height than the "nutritional" children $(\boldsymbol{\Lambda})$, but that also the points for the "control" groups are mainly to the left of those for the "nutritional" groups. This shows that, even when the height gains are the same for the two groups, the "controls" gain more weight, or alternatively that the children of the "nutritional" groups require a greater height gain than those in the "control" groups in order that their weight gain should be the same.

These results confirm the findings shown above that weight growth is affected by nutritional conditions more than height growth.
Growth DURING School Holidays.-The Newman regime operates only during term time* and it is interesting to compare the growth during the summer holiday months of July and August. This averaged from about one-fifth to one-quarter of the year's growth (Table VI, opposite).

The "siblings" gained the biggest proportion of their annual weight increase, whilst the Newman girls gained less than the normal proportion and the Newman boys were about average.

*There was a shorter summer break than in other schools and a school camp was organized, but the growth was compared for the same period. 
TABLE VI

GROWTH DURING SUMMER HOLIDAY AS PERCENTAGE OF ANNUAL WEIGHT GAIN

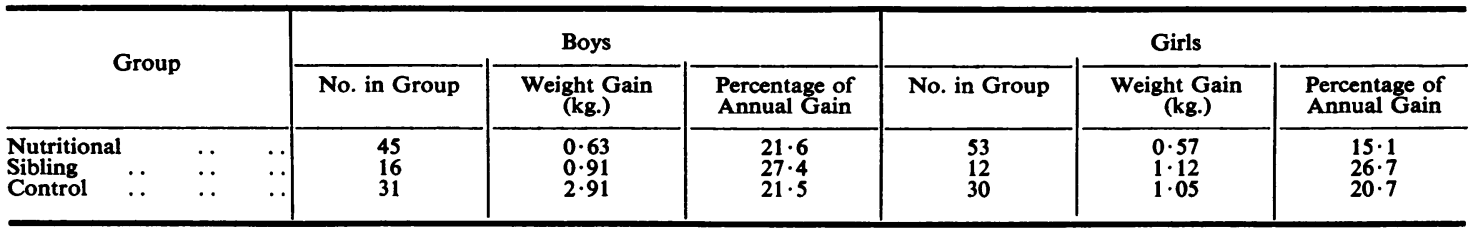

TABLE VII

MEAN WEIGHT AND HEIGHT GAINS OF CHILDREN STILL AT THE NEWMAN SCHOOL COMPARED WITH THOSE WHO LEFT

\begin{tabular}{|c|c|c|c|c|c|c|c|c|c|c|c|c|}
\hline \multirow{3}{*}{$\begin{array}{l}\text { Age } \\
\text { (yrs) }\end{array}$} & \multicolumn{6}{|c|}{ Present Newman Pupils (Debilitated) } & \multicolumn{6}{|c|}{ Former Newman Pupils } \\
\hline & \multicolumn{4}{|c|}{ Weight Gain (kg.) } & \multicolumn{2}{|c|}{ Height Gain (cm.) } & \multicolumn{4}{|c|}{ Weight Gain (kg.) } & \multicolumn{2}{|c|}{ Height Gain (cm.) } \\
\hline & No. & $\begin{array}{l}\text { Boys } \\
\text { Gain }\end{array}$ & No. & $\begin{array}{l}\text { Girls } \\
\text { Gain }\end{array}$ & Boys & Girls & No. & $\begin{array}{l}\text { Boys } \\
\text { Gain }\end{array}$ & No. & $\begin{array}{l}\text { Girls } \\
\text { Gain }\end{array}$ & Boys & Girls \\
\hline $\begin{array}{r}9 \\
10 \\
11 \\
12 \\
13 \\
14 \\
15\end{array}$ & $\begin{array}{r}18 \\
17 \\
13 \\
10 \\
11 \\
5 \\
-\end{array}$ & $\begin{array}{l}2 \cdot 2 \\
2 \cdot 6 \\
3 \cdot 3 \\
3 \cdot 6 \\
4 \cdot 5 \\
5 \cdot 8 \\
-\end{array}$ & $\begin{array}{r}14 \\
18 \\
24 \\
21 \\
13 \\
5 \\
2\end{array}$ & $\begin{array}{l}2 \cdot 9 \\
2 \cdot 8 \\
4 \cdot 0 \\
4 \cdot 7 \\
4 \cdot 7 \\
2 \cdot 5 \\
3 \cdot 8\end{array}$ & $\begin{array}{l}5 \cdot 3 \\
4 \cdot 6 \\
5 \cdot 2 \\
6 \cdot 9 \\
6 \cdot 7 \\
7 \cdot 8 \\
8 \cdot 0\end{array}$ & $\begin{array}{l}5 \cdot 2 \\
6 \cdot 4 \\
6 \cdot 9 \\
6 \cdot 3 \\
5 \cdot 2 \\
3 \cdot 0 \\
3 \cdot 3\end{array}$ & $\begin{array}{l}1 \\
3 \\
3 \\
2 \\
2 \\
\overline{1}\end{array}$ & $\begin{array}{l}1 \cdot 4 \\
2 \cdot 1 \\
2 \cdot 9 \\
3 \cdot 1 \\
4 \cdot 8 \\
-5 \cdot 6\end{array}$ & $\begin{array}{l}2 \\
1 \\
3 \\
6 \\
5 \\
-1\end{array}$ & $\begin{array}{l}1 \cdot 7 \\
2 \cdot 8 \\
2 \cdot 8 \\
4 \cdot 9 \\
4 \cdot 8 \\
\overline{0 \cdot 2}\end{array}$ & $\begin{array}{l}4 \cdot 5 \\
4 \cdot 6 \\
6 \cdot 3 \\
6 \cdot 5 \\
7 \cdot 3 \\
\overline{7 \cdot 3}\end{array}$ & $\begin{array}{l}5 \cdot 0 \\
4 \cdot 0 \\
5 \cdot 9 \\
6 \cdot 0 \\
3 \cdot 4 \\
-1 \cdot 1\end{array}$ \\
\hline
\end{tabular}

These results suggest that the children in the "nutritional" groups would probably have fared unfavourably compared with their siblings if they had not been receiving the Newman School treatment. This is confirmed by an analysis of the annual growth in weight and height of the Newman children who left the school when they were considered fit enough to attend ordinary schools, compared with that of the children still remaining (Table VII)

Although the numbers are small, at practically all ages the children who left the Newman school failed to grow as much as those remaining, despite the fact that they were no longer considered to be debilitated.

School AtTendance.-In addition to growth and the other physical criteria of health, illness records afford the most direct information on whether conditions are adequate or not. Full health and absence records were kept at the Newman School, but it was not possible to obtain such detailed health records for the control group. Nevertheless, each child was seen monthly and any medical attention received was noted. Overall attendance figures for the Newman and control children still in the study in 1954 for the 3 years during which the study had been in progress are shown in Table VIII.

The figures include all causes of absence, and probably under-estimate the difference between children in the "nutritional" and "control" groups. As there was some pressure for places there was a greater incentive for the Newman school children to
TABLE VIII

REI.ATION BETWEEN GAINS IN WEIGHT AND HEIGHT

\begin{tabular}{|c|c|c|c|c|c|c|c|c|}
\hline \multirow[b]{3}{*}{ School } & \multicolumn{4}{|c|}{ Newman } & \multicolumn{4}{|c|}{ Control } \\
\hline & \multicolumn{2}{|c|}{ Boys } & \multicolumn{2}{|c|}{ Girls } & \multicolumn{2}{|c|}{ Boys } & \multicolumn{2}{|c|}{ Girls } \\
\hline & No. & $\begin{array}{c}\text { Mean } \\
\text { Per- } \\
\text { centage } \\
\text { Atten- } \\
\text { dance }\end{array}$ & No. & $\begin{array}{c}\text { Mean } \\
\text { Per- } \\
\text { centage } \\
\text { Atten- } \\
\text { dance }\end{array}$ & No. & $\begin{array}{c}\text { Mean } \\
\text { Per- } \\
\text { centage } \\
\text { Atten- } \\
\text { dance }\end{array}$ & No. & $\begin{array}{c}\text { Mean } \\
\text { Per- } \\
\text { centage } \\
\text { Atten- } \\
\text { dance }\end{array}$ \\
\hline $\begin{array}{l}\text { Junior } \\
\text { Senior }\end{array}$ & $\begin{array}{r}18 \\
8\end{array}$ & $\begin{array}{l}87 \cdot 9 \\
87 \cdot 8\end{array}$ & $\begin{array}{l}11 \\
17\end{array}$ & $\begin{array}{l}83 \cdot 9 \\
86 \cdot 8\end{array}$ & $\begin{array}{l}14 \\
15\end{array}$ & $\begin{array}{l}95 \cdot 1 \\
96 \cdot 0\end{array}$ & $\begin{array}{l}15 \\
21\end{array}$ & $\begin{array}{l}94 \cdot 5 \\
91 \cdot 8\end{array}$ \\
\hline
\end{tabular}

attend regularly except when prevented by genuine ill-health, lest they should lose their place at the school. Further, a bus picked up the Newman children from their homes, so that they were less likely to stay away on account of bad weather than those attending other schools. The figures show a very good health record for the controls and a less good one for the "nutritional" group.

Within-school correlations between attendance and physical criteria were calculated for both boys and girls in order to see whether there was any association apart from that shown by the generally higher attendance and superior physical characteristics of the control children compared with the "nutritional" group. (Only 6 per cent. of control children had as much absence as the average for the Newman school children and only 10 per cent. had as low attained weight or weight-for-height.) However, the correlations were not significantly different from zero, and 
within the same schools, therefore, the children with most absence did not differ in physique from the other children.

Home Conditions.-As already remarked, the Newman School regime could only operate during term time and then only for part of the day and, therefore, home conditions could still affect the children's progress. Assessments of the economic status and household management of the home were made for the Newman schoolchildren by the School Nurse. Table IX shows that absence from school decreased as the home conditions improved.

TABLE IX

PERCENTAGE ATTENDANCE ACCORDING TO ECONOMIC STATUS AND HOUSEHOLD MANAGEMENT

\begin{tabular}{|c|c|c|c|c|c|c|}
\hline \multirow{2}{*}{ Grade } & \multicolumn{2}{|r|}{ Plus } & \multicolumn{2}{|r|}{ Average } & \multicolumn{2}{|r|}{ Minus } \\
\hline & No. & $\begin{array}{l}\text { Percentage } \\
\text { Attendance }\end{array}$ & No. & $\begin{array}{l}\text { Percentage } \\
\text { Attendance }\end{array}$ & No. & $\begin{array}{l}\text { Percentage } \\
\text { Attendance }\end{array}$ \\
\hline $\begin{array}{l}\text { Economic } \\
\text { Status } \quad \ldots\end{array}$ & 9 & 91 & 33 & 85 & 11 & 88 \\
\hline $\begin{array}{l}\text { Household } \\
\text { Management }\end{array}$ & 11 & 92 & 24 & 88 & 18 & 85 \\
\hline
\end{tabular}

The relations of economic status and household management to the physical development of the children in the "nutritional" group at the end of the study are shown in Table $\mathrm{X}$. For simplicity the results are expressed as deviations from age norms.

Besides the generally low level of physique in the "nutritional" group, there were gradients in most characteristics according to economic conditions and household management, even within the narrow limits of home conditions found in the group. Berry and Cowin (1954) found that household management ratings, mothers' intelligence, and sleeping conditions, as well as dietary differences all affected children's physical measurements.

\section{Discussion}

The attained measurements of the three groups remained very much the same in relation to age throughout the study. This indicates that, even when the Newman children were considered healthy enough to take their place within the ordinary schools, they had not attained the physique of the controls or, in fact, of normal average children. In addition to the nutritional differences there may, therefore, be some other differences, e.g. in the hereditary make-up of the groups which affected selection. When the weights and heights of the available parents of the children in the "nutritional" group were measured, they were found to be significantly shorter but not lighter than the national averages for the corresponding ages.

This somewhat slender evidence and the smallerthan-average size of the siblings (who were considered to be in normal health), suggests that there may be constitutional differences between the groups.

Even in respect of weight for height or weight according to skeletal measurements (which allow a lower standard) the children in the "nutritional" group had not quite reached normal. It may, therefore, be unjustifiable to expect these children to have the same gain in weight and height as the controlswho were not only bigger but often above average for age.

During the school holidays when the living conditions can be presumed to be similar, the growth of the "nutritional" group appeared to fall behind that of the "siblings". This suggests that the open air school was maintaining the "nutritional" group at a higher level than they would otherwise have reached. Follow-up results showed that after leaving the Newman School the children tended to regress.

It is also noteworthy that the Newman School

TABLE $X$

PHYSICAL CRITERIA ACCORDING TO ECONOMIC STATUS AND HOUSEHOLD MANAGEMENT (NUTRITIONAL GROUP ONLY) EXPRESSED AS DEVIATIONS ABOUT AGE STANDARDS

\begin{tabular}{|c|c|c|c|c|c|c|c|c|}
\hline \multirow{2}{*}{ Grade } & \multirow{2}{*}{ 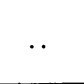 } & \multirow{2}{*}{ * } & \multicolumn{3}{|c|}{ Household Management } & \multicolumn{3}{|c|}{ Economic Status } \\
\hline & & & Plus & Average & Minus & Plus & Average & Minus \\
\hline No. of Children & $\cdots$ & .. & 21 & 12 & 22 & 8 & 32 & 15 \\
\hline \multirow{6}{*}{$\begin{array}{l}\text { Physical } \\
\text { Criteria }\end{array}$} & Weight (kg.) & $\cdots$ & $-5 \cdot 8$ & $-8 \cdot 1$ & $-6 \cdot 4$ & $-4 \cdot 27$ & $-6 \cdot 27$ & $-8 \cdot 31$ \\
\hline & Height (cm.) & $\begin{array}{ll}\cdots & \cdots \\
\end{array}$ & $-1 \cdot 0$ & $-15 \cdot 6$ & $-5 \cdot 6$ & $5 \cdot 0$ & $-4 \cdot 0$ & $-5 \cdot 3$ \\
\hline & \multicolumn{2}{|c|}{$\begin{array}{l}\text { Weight minus } \\
\text { Predicted Weight (kg.) }\end{array}$} & -0.55 & $-1 \cdot 18$ & $-1 \cdot 00$ & 0.09 & -2.05 & -3.09 \\
\hline & \multicolumn{2}{|c|}{ Body Fat $(0.1 \mathrm{~mm})}$. & $-7 \cdot 9$ & $-17 \cdot 3$ & $-15 \cdot 6$ & $-5 \cdot 0$ & $-13 \cdot 5$ & $-16 \cdot 3$ \\
\hline & \multicolumn{2}{|c|}{ Weight Gain (kg.) } & -0.41 & -0.36 & $-0 \cdot 27$ & $0 \cdot 32$ & $-1 \cdot 32$ & $-2 \cdot 18$ \\
\hline & \multicolumn{2}{|c|}{ Height Gain (cm.) } & $+1 \cdot 4$ & $0 \cdot 5$ & 0.9 & $2 \cdot 0$ & $3 \cdot 3$ & $+2 \cdot 1$ \\
\hline
\end{tabular}


regime was unable to remove differences associated with the poorer home conditions.

These findings suggest that further progress might be made by ensuring that the home conditions (especially of feeding and sleeping) are maintained at an adequate level.*

It might also be worth considering extending the accommodation to full residence at least for part of the year. The data certainly do not support any suggestion that the length of treatment could be reduced without adversely affecting its results. Practical considerations of demand for accommodation in the Newman School may even already require that some children may have to leave before the full benefit to their physique has been achieved.

\section{SUMmaRY}

(1) The investigation compares the physique and growth of debilitated children at an Open Air School with their brothers and sisters attending ordinary schools and with a control group of selected healthy children over a period of 3 years.

(2) Weights and heights, chest girth, body fat, acromial breadth, and "ideal" weights predicted from skeletal measurements were all found to be low in the "nutritional" debility and "sibling" groups compared with standards based on the physique of L.C.C. children or with means for age. Much of the nutritional group's inferiority in physique was of long standing.

(3) Growth during the period was also greater for the controls than for the other groups, although

*Attempts were made to see that the parents of the Newman children gave them the ordinary meals provided for the rest of the family and did not rely on the Newman School meals as being sufficient in themselves. the girls in the "nutritional" group increased most in the last year. Height gains showed smaller differences.

(4) Growth during school holidays and after leaving the Newman School were especially low in the "nutritional" group.

(5) Home circumstances appeared to affect the progress of those at the Newman School, in spite of the better school conditions.

(6) Normal growth and physique were not reached by most of the Newman children even when they left the school, but they appeared to maintain a higher level than they would have reached without the special treatment.

We wish to express our thanks to Dr. E. R. Bransby of the Ministry of Health for initiating this study, and to all those who co-operated in carrying out the project; especially to the late Mr. W. Child, Headmaster of the Newman School, for his enthusiastic help throughout, and to the other head teachers, Miss B. Greenhalgh, Mr. J. H. Bagot and Mr. G. Naylor. We are also grateful to Nurse G. K. Cave of the Newman School, who made the home assessments, and who took, with Nurse N. Lloyd, the monthly measurements, and to Miss W. M. Cooper of the office staff for record keeping and clerical help.

\section{REFERENCES}

Berry, W. T. C., and Cowin, P. J. (1954). Brit. med. J., 1, 847.

Bransby, E. R., and Hammond, W. H. (1950). Proc. roy. Soc. Med., 43, 825.

- (1951), Brit. med. J., 2, 330.

Hammond, W. H. (1953a). British Journal of Preventive and Social Medicine, 7, 231.

—_ (1953b). Hum. Biol., 25, 65.

- (1955). British Journal of Preventive and Social Medicine, 9, 201. Daley, A. (1950). "L.C.C. Weights and Heights for 1949". L.C.C. 